\title{
PENSAMIENTO CONSTRUCTIVO BAJO LA VISIÓN DE LA INTELIGENCIA EMOCIONAL EN ESTUDIANTES UNIVERSITARIOS
}

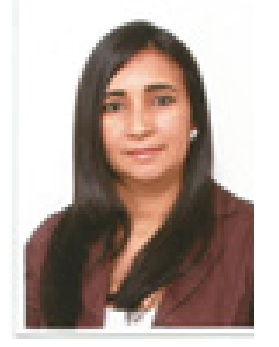

\author{
Sonia Ferrer Planchart \\ Universidad del Zulia Núcleo COL, Venezuela \\ Soniaferrerp4053@gmail.com
}

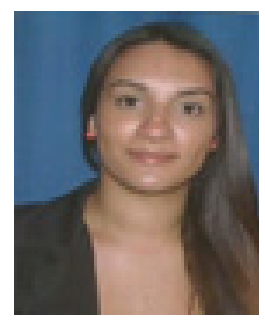

\section{Estefany Elinett Caridad Ferrer \\ Universidad Pedagógica Experimental Libertador, Venezuela elinet.estefany@gmail.com}

\section{RESUMEN}

El objetivo general de la presente investigación es determinar el pensamiento constructivo bajo la visión de la inteligencia emocional en los estudiantes universitarios de nuevo ingreso del núcleo LUZ COL. Teóricamente se sustentó en los planteamientos de Epstein (1998), Mayer y Salovey, (1990), Garaigordobil y Oñederra (2010). Bisquerra (2011) Chávez, Contreras, y Velásquez (2014).

La metodología se desarrolló desde un enfoque positivista, el tipo de investigación es descriptiva. La población está conformada por los estudiantes de primer ingreso del núcleo LUZ COL. Como técnica de recolección de la información, se utilizó la encuesta, y como instrumento, el cuestionario sobre pensamiento constructivo diseñado por Epstein en 1987 que presenta una confiabilidad Alfa Cronbach de 0,833 lo que indica su validez para utilizarlo en el presente estudio.

Los resultados obtenidos reportaron que los estudiantes de nuevo ingreso del núcleo LUZCOL tienen una mediana presencia en lo que corresponde al pensamiento constructivo es importante destacar que en las dos primeras escalas sobre emotividad y eficacia presentaron promedios altos que indican que se aceptan a sí mismos y a los demás, también actúan con decisión en situaciones controlables y con escasa ansiedad en situaciones poco controlables. 
y con escasa ansiedad en situaciones poco controlables. Pero en lo que concierne a las escalas de pensamiento rígido se obtuvo una mediana presencia y una alta presencia relacionada con el pensamiento ingenuo lo que indica ciertos pensamientos desadaptativos y racionales con creencias básicas que determinan un modo de actuar sobre prejuicios e intolerancia así como la percepción de la vida de una manera simplista.

Palabras clave: Pensamiento constructivo, inteligencia emocional, estudiantes universitarios, escalas de afrontamiento, desadaptativos, adaptativos.

\title{
CONSTRUCTIVE THINKING UNDER THE VISION OF EMOTIONAL INTELLIGENCE IN UNIVERSITY STUDENTS
}

\begin{abstract}
The main objective of this paper is to determine constructive thinking under the vision of emotional intelligence among the University of Zulia sophomore students. It is theoretically based on the approaches of Epstein (1998), Mayer and Salovey, (1990), Garaigordobil and Onederra (2010). Bisquerra (2011) Contreras, Chavez, and Velasquez (2014).

The methodology was developed from a positivist approach, the type of research is descriptive. The population was composed of sophomore students at the University of Zulia, Cabimas campus. Survey was used as technique for data collection, and as for instrument, a questionnaire on Constructive Thinking, designed by Epstein in 1987 that presents an Alpha Combrach reliability of 0,8333 which in turn indicates its reliability to be used in this study.

The results showed that sophomores students have a medium presence as for Constructive Thinking, it is important to highlight that in the first two scales on emotivity and efficiency showed higher mean which indicates that they accept themselves and others, they also act with determination in manageable situations and with low anxiety in less manageable situations. However, as for scales of rigid thinking, a medium presence was obtained and a higher presence related with naïve thinking which indicates certain unadaptive and reactive thinking with basic believes that determine a manner of behaving on prejudice and intolerance, as well as the perception of a more simple life.
\end{abstract}

Key words: Constructive Thinking, University students, facing scales, unadaptative, adaptatives. 
Pensamiento constructivo bajo la visión de la inteligencia emocional en estudiantes universitarios

Sonia Ferrer Planchart - Estefany Elinett Caridad Ferrer (28:41)

\section{INTRODUCCIÓN}

El pensamiento constructivo es un concepto teórico que hace referencia a esquemas de percepción, emoción y acción sobre la representación que hacen las personas de su realidad. Los componentes de este constructo son la inteligencia afectiva y la inteligencia práctica. (Epstein, 1998). Este sistema de procesamiento de la información actúa de manera paralela al sistema racional y tiene sus orígenes en las experiencias de la niñez, éstas altamente cargadas de emociones; en este proceso se da una asociación entre la emoción y los eventos vividos, apartando la lógica como sucede en el sistema racional.

De manera que la percepción, la comprensión y la regulación emocional son elementos necesarios para lograr una adaptación al ambiente y a las circunstancias negativas que rodean a la persona, también influye de manera importante el potencial intelectual que se posee al momento de encontrar las soluciones a determinados problemas.

En este sentido se considera que las personas con alto pensamiento constructivo tienen altas probabilidades de adaptarse a las situaciones y de tener éxito en el logro de sus metas. Por el contrario las personas con un pensamiento poco constructivo, o poco inteligente emocionalmente tienden a percibir el mundo de manera negativa, les cuesta adaptarse, además de seleccionar estrategias inadecuadas para solucionar los problemas, también presentan una autoestima baja que los puede llevar a la depresión y desesperanza. (Martínez-Otero, 2002).

En el sistema de procesamiento experiencial de la realidad como también se le conoce tiene lugar relevante la manera como las personas se perciben así mismas (sus potencialidades) al mundo (como se comprende la problemática actual y que hacemos frente a ella) y al futuro. Este tipo de pensamiento impactará de manera directa su desarrollo en el contexto personal, social y académico de la persona.

No obstante, en el ámbito académico los estudiantes que tienen poco pensamiento constructivo, no logran desarrollar todo el potencial indicado por su coeficiente intelectual, pareciera que su capacidad de pensar constructivamente interfiere con su capacidad intelectual. En el caso de un estudiante que no logra relacionarse adecuadamente con sus profesores y compañeros, y exhibe una confianza excesiva o una actitud derrotista, o que se agota psíquica e incluso físicamente por el estrés, realizará con desventajas sus estudios por lo tanto su rendimiento académico será bajo. (Contreras, Chávez, Aragón, y Velásquez 2010, Chávez, Contreras y Velásquez, 2014).

Se entiende entonces la importancia de manejar un pensamiento constructivo como fuente de inteligencia emocional en el caso de los estudiantes de primer ingreso quienes se enfrentarán a un nuevo contexto educativo que incluye no solo diferente estructura física sino cambios en los 
planes de estudios, profesores, procesos administrativos, sistemas de evaluación y grupos de amigos; todo ello relacionado además con un ambiente con problemas políticos, económicos, familiares, o de salud en los que están expuestos no sólo los jóvenes Venezolanos sino de América latina y del mundo; circunstancias generadoras para muchos estudiantes de posible estrés que se convierte en desánimo y baja motivación hacia los estudios por las dificultades naturales que se presentan en la vida, lo que pone a prueba constantemente sus pensamientos e inteligencia emocional para enfrentar dichas situaciones.

Es importante destacar que en el caso de Venezuela no existen investigaciones previas publicadas que se relacionen con este tema por ello se considera un estudio relevante con aportes significativos para profundizar en el abordaje de estrategias que potencien el pensamiento constructivo en el estudiante universitario de nuevo ingreso.

Por las explicaciones anteriores el presente artículo tiene como objetivo determinar el pensamiento constructivo bajo la visión de la inteligencia emocional en los estudiantes universitarios de nuevo ingreso del núcleo LUZ COL.

\section{FUNDAMENTOS TEÓRICOS}

El concepto de inteligencia emocional en esta década ha adquirido gran relevancia y popularidad que ha llevado este tema a ser el centro de múltiples investigaciones tanto en la psicología, la educación así como el contexto organizacional. La importancia de la inteligencia emocional radica en aprender a manejar las emociones para que éstas actúen a favor de la persona. (Mayer y Salovey, 1990, Garaigordobil y Oñederra 2010).

Sobre este tema Bisquerra (2011) plantea que tener una conciencia emocional consiste en conocer las propias emociones y las emociones de los demás, esto se logra a través de la propia observación y la observación de las personas que nos rodean. Culmina el autor afirmando que "aprender a regular las emociones (ira, rabia, enfado, ansiedad, estrés, tristeza) en situaciones críticas es esencial para las buenas relaciones interpersonales y para el bienestar personal y social".

Como estrategia a la conciencia emocional se presenta la autonomía emocional que es un paso a la regulación que incluye la importancia de la autoestima, la autoeficacia y la automotivación; se trata de formarse para conseguir el equilibrio, asertividad y resiliencia ante los problemas personales y sociales.

Se entiende entonces que la persona que tiene conocimiento de sus fortalezas y debilidades es capaz de valorar positiva o negativamente los sucesos de la vida, para ello sus creencias y pensamientos son elementos importantes para enfrentar las diversas situaciones y poder adaptarse 
Pensamiento constructivo bajo la visión de la inteligencia emocional en estudiantes universitarios

Sonia Ferrer Planchart - Estefany Elinett Caridad Ferrer (28:41)

a las circunstancias inesperadas de manera positiva.

Una importante contribución en este campo del estudio de las emociones, las creencias y los pensamientos es la teoría cognitivo- experiencial propuesta por Epstein (1998) quien enfoca su estudio en la evaluación de las creencias básicas, de carácter preconsciente que orienta la percepción que las personas tienen acerca de los eventos de la vida cotidiana, así como de las emociones que estas situaciones resultan en ellas condicionándolos a actuar con ciertos patrones de comportamientos. Esta teoría refiere que las emociones que se experimentan en la vida diaria están producidas por la interpretación que realiza la persona de su realidad.

\section{La teoría cognitiva experiencial}

La teoría cognitivo experiencial de Epstein (2003) es una teoría integral de la personalidad que se relaciona con otras teorías psicodinámicas así como las teorías del aprendizaje y el enfoque del procesamiento de la información.

Esta teoría establece un paralelismo entre el inconsciente freudiano mal adaptativo y el sistema experiencial adaptativo. Al mismo tiempo se rige bajo tres principios fundamentales:

- Las personas procesan la información a través de dos sistemas conceptuales independientes: el sistema experiencial preconsciente y el sistema racional consciente.

- El sistema experiencial está regido por la emoción.

- Asume que el ser humano posee cuatro necesidades básicas como la búsqueda de placer, de relacionarse, de mantener la estabilidad y el mantenimiento de la autoestima.

El comportamiento está definido por la influencia de las necesidades que sean activadas en cada situación, éstas se equilibran entre sí con el fin de lograr la motivación y la satisfacción de las mismas. Las personas que no poseen salud mental tienden satisfacer sus necesidades de modo conflictivo, mientras que las personas sanas mentalmente lo hacen de modo equilibrado, permitiéndoles mantener un sistema racional conceptual estable; es decir pueden lograr un balance favorable entre las situaciones placenteras y las de dolor que les permite mantener relaciones interpersonales satisfactorias y un alto nivel de autoestima.

De acuerdo con esta teoría, los seres humanos responden mediante dos sistemas, el experiencial y el racional. El sistema experiencial se presenta en los seres humanos como una manera de adaptarse al medio, esta adaptación surge mediante el aprendizaje por experiencia más que por deducción lógica, contexto perteneciente al sistema racional.

El modo como opera el sistema experiencial es inconsciente, automático, rápido, concreto sin esfuerzo con pocos recursos cognitivos. 
En este proceso se codifica la información de dos modos: memoria de eventos individuales sobre todo aquellos que poseen un alto grado de emoción y otro modo más general formado por representaciones o metáforas. Se comprende entonces que este sistema es influenciado por el afecto ante una situación que vive la persona por un evento con contenido emocional que logra traer a su memoria. Estos recuerdos traídos a la memoria además de los sentimientos guían el procesamiento del acontecimiento y el comportamiento de la persona ante el mismo.

Dependiendo si el contenido de esos recuerdos es positivo o negativo, la persona tratará de guiar su conducta hacia la situación para reproducir esos sentimientos o rechazarlos para no experimentar de nuevo sentimientos negativos. Esta valoración de la realidad es inconsciente aunque se trate de conseguir explicaciones mediante el sistema racional para comprender las actuaciones de las personas. (Epstein 2003).

El sistema racional es un sistema inferencial que opera de acuerdo con lo que la persona entiende sobre la realidad culturalmente transmitida, éste opera de manera consciente, analítica, requiere esfuerzo, no es afectivo y necesita altos niveles de recursos cognitivos. Este sistema puede entender el funcionamiento del sistema experiencial pero no al contrario.

El sistema experiencial nos permite responder adaptativamente a los problemas de la vida mediante un análisis integral sin necesidad de analizar los componentes de la situación.

\section{EI Pensamiento constructivo}

Epstein (1998) quien ha señalado el término pensamiento constructivo dentro de su teoría considera la existencia de dos pensamientos: El afrontamiento experiencial que es la inteligencia relacionada con el buen manejo de las emociones negativas, y el afrontamiento conductual que es un pensamiento orientado a la acción, aceptar los retos y pensar de una manera eficaz de solucionar los problemas. Una persona constructiva alcanza un equilibrio y niveles elevados de ambos pensamientos.

Así que para este autor el pensamiento constructivo se refiere a la capacidad de relacionarnos con los demás de un modo efectivo y de forma satisfactoria (inteligencia emocional) solucionar problemas interpersonales de una manera adecuada (inteligencia social) y la capacidad para solucionar eficazmente los problemas que surgen de la vida diaria (inteligencia práctica) (Chávez, Contreras y Velásquez 2014).

De esta manera el pensamiento constructivo es un concepto teórico que hace referencia a la capacidad de reconocer las propias emociones y sentimientos, además de la regulación y dirección consciente que de estas emociones se haga para solucionar los problemas, así como para el logro 
Pensamiento constructivo bajo la visión de la inteligencia emocional en estudiantes universitarios

Sonia Ferrer Planchart - Estefany Elinett Caridad Ferrer (28:41)

de las metas que cada persona se proponga con el mayor nivel de satisfacción personal menor estrés y adaptación con el medio social donde se desenvuelva.

\section{Características de las personas con pensamiento constructivo y pensamiento poco constructivo}

Las personas con alto pensamiento constructivo tienen más posibilidades de adaptarse a las situaciones y de obtener éxitos en sus proyectos, en cambio las personas con poca inteligencia emocional tienden a percibirse a sí mismo y al mundo de una manera negativa, los que los predispone a actuar de manera poco adaptativa teniendo como resultado la elección de estrategias inadecuadas para la solución de los problemas, en algunos casos por su auto concepto y auto eficacia débil los pueden llevar a la depresión y desesperanza.

\section{METODOLOGIA}

Tomando en cuenta que el objetivo general del presente artículo es determinar el pensamiento constructivo bajo la visión de la inteligencia emocional en los estudiantes universitarios de nuevo ingreso del núcleo LUZ COL. Se consideró que el tipo de investigación es descriptiva. Al respecto, Hernández, Fernández y Baptista, (2014 p. 60), afirman que "los estudios descriptivos buscan especificar las propiedades importantes de personas, grupos o cualquier otro fenómeno que sea sometido a análisis". El diseño es no experimental transversal por cuanto de acuerdo a Hernández, Fernández, y Baptista (2014 p.184) “se realiza sin manipular deliberadamente variables" en un momento único durante el proceso, sin pretender medir la evolución del fenómeno estudiado.

Los sujetos de estudio que conforman la presente investigación los define Hurtado de Barrera (2010, p. 267) como "unidades de estudio pertenecientes al evento que se va a estudiar". La población está conformada por los estudiantes de primer ingreso cursantes del periodo académico 1-2016. En la siguiente tabla se presenta la distribución de estudiantes por carrera pertenecientes al Núcleo LUZ-COL.

\begin{tabular}{|c|c|c|}
\hline PROGRAMA & CARRERA & $\begin{array}{c}\text { NÙMERO DE } \\
\text { ESTUDIANTES }\end{array}$ \\
\hline \multirow{4}{*}{$\begin{array}{c}\text { Humanidades y } \\
\text { Educación }\end{array}$} & Educación Especial & 120 \\
\hline & $\begin{array}{c}\text { Educación Industrial } \\
\text { Área Mecánica }\end{array}$ & 380 \\
\hline & Educación Informática & 180 \\
\hline & $\begin{array}{c}\text { Educación Industrial } \\
\text { Electricidad }\end{array}$ & 115 \\
\hline \multirow{4}{*}{$\begin{array}{c}\text { Ciencias } \\
\text { Económicas y } \\
\text { Sociales }\end{array}$} & Economia & 110 \\
\hline & Administración & 450 \\
\hline & Relaciones Industriales & 750 \\
\hline & Contaduría Pública & 606 \\
\hline \multirow[b]{4}{*}{ Ingeniería } & Ingeniería Mecánica & 1185 \\
\hline & Ingenieria de Petróleo & 959 \\
\hline & Ingeniería Civil & 680 \\
\hline & Ingeniería Ambiental & 228 \\
\hline & Total & 5763 \\
\hline
\end{tabular}


Según, Pallela y Martins (2010, p. 116) la muestra "no es más que la escogencia de una parte representativa de una población, cuyas características reproduce de la manera más exacta posible". En la selección de la muestra de la presente investigación, se seleccionó el muestreo no probabilístico en el cuál los sujetos de la muestra no se seleccionan al azar sino que la escogencia se realiza tomando en cuenta algunos criterios establecidos por el investigador, también se tomó en cuenta la proximidad al momento de seleccionar la muestra, para ello se recurrió a la técnica del muestreo accidental por ser un procedimiento práctico que utiliza el investigador cuando las muestras son cercanas a su estudio.

En este caso se seleccionó el programa de ingeniería por poseer mayor unidades de muestras homogéneas entre sí. (Ander-Egg 2004, p.363).

Tabla 2 Distribución de la muestra estudiantes de nuevo ingreso del Programa de Ingeniería

\begin{tabular}{|c|c|c|}
\hline \multirow{4}{*}{ Ingeniería } & Ingeniería Mecánica & 1185 \\
\cline { 2 - 3 } & Ingeniería de Petróleo & 959 \\
\cline { 2 - 3 } & Ingeniería Civil & 680 \\
\cline { 2 - 3 } & Ingenieria Ambiental & 228 \\
\cline { 2 - 3 } & Total & 3052 \\
\hline
\end{tabular}

Fuente: Elaboración propia (2016)

Ahora bien, como se refirió anteriormente la población corresponde a los estudiantes de nuevo ingreso del Programa de Ingeniería del Núcleo LUZ-COL, tomando un grupo conformado por 3052 estudiantes, calculándose la muestra mediante la fórmula de Sierra (2005) para poblaciones finitas entre (1 y 100.000) quedando la muestra en 97 estudiantes.

Tabla 3

Distribución de la muestra por sexo

\begin{tabular}{|l|l|c|c|}
\hline \multicolumn{2}{|c|}{} & Frecuencia & Porcentaje \\
\hline Válido & $\begin{array}{l}\text { Masculin } \\
\text { o }\end{array}$ & 53 & 54,6 \\
\cline { 2 - 4 } & $\begin{array}{l}\text { Femenin } \\
\text { o }\end{array}$ & 44 & 45,4 \\
\cline { 2 - 4 } & Total & 97 & 100 \\
\hline
\end{tabular}

Fuente: Elaboración propia (2016)

En cuanto a los instrumentos de recolección de datos éstas "comprenden procedimientos y actividades que le permiten al investigador obtener la información necesaria para dar respuesta a la pregunta de investigación" (Hurtado de Barrera 2010, p. 271).

La recolección de datos se realizó con el cuestionario que es definido por Hernández, Fernández, y Baptista, (2014, p.276) como "un conjunto de preguntas respecto a una o más variables a medir", para lo cual se utilizó un instrumento definido como: Inventario de Pensamiento Constructivo (CTI) elaborado por Seymor Epstein en 1987 y adaptado por el departamento de Investigación y Desarrollo de TEA Ediciones S.A. (2012). 
Pensamiento constructivo bajo la visión de la inteligencia emocional en estudiantes universitarios

Sonia Ferrer Planchart - Estefany Elinett Caridad Ferrer (28:41)

El área de pensamiento constructivo posee dos escalas: Afrontamiento emocional y afrontamiento conductual que a su vez integran las sub escalas, emotividad subdividida por los indicadores: Autoestima, tolerancia, impasibilidad, capacidad de sobreponerse, posee 15 items, Eficacia subdividida por los indicadores: Pensamiento positivo, actividad y responsabilidad, contiene 16 items. Por otro lado el pensamiento no constructivo consta de cuatro escalas pensamiento supersticioso esta posee 13 items, rigidez con los sub indicadores: extremismo, suspicacia, e intolerancia con 29 items, pensamiento esotérico con los indicadores creencias paranormarles e irracionales con 14 items y pensamiento ingenuo con los indicadores: euforia, pensamiento estereotipado e ingenuidad con 21 indicadores.

Se trata de una medida de auto informe para adolescentes y adultos. Este Inventario consta de 108 items que se puntúan en escala tipo Likert de 5 puntos en función del grado de acuerdo o desacuerdo con cada una de las afirmaciones. Ha sido elaborada para evaluar el pensamiento constructivo y destructivo que se produce de manera automática en la vida diaria.

Dicho instrumento contiene una dimensión general (PGC) y seis escalas que, a su vez, tienen varias dimensiones, con las que pretende evaluar el pensamiento constructivo o inteligencia experiencial, el cual se relaciona con varias facetas de la inteligencia emocional. El instrumento sobre pensamiento constructivo diseñado por Epstein en 1987 presenta una confiabilidad Alfa Cronbach de 0,833 lo que indica su validez para utilizarlo en el presente estudio.

Una vez aplicados los instrumentos a la población en estudio se procedió a su codificación y tabulación utilizando para ello el paquete estadístico SPSS. Los resultados serán procesados mediante la aplicación de la estadística descriptiva a través de la medida de tendencia central (media) el análisis de frecuencias (absolutas y porcentuales), y la desviación típica, datos que se procesaran tomando en cuenta el baremo para interpretar dichos datos.

\section{ANÁLISIS DE LOS RESULTADOS}

El análisis de los resultados se realizó mediante la organización de los datos para dar respuesta al objetivo de la investigación en el cual se plantea determinar si los estudiantes de ingeniería de primer ingreso del núcleo LUZ-COL poseen un pensamiento constructivo que les permita desarrollar todo su potencial en el proceso de su carrera y su vida universitaria, en la tabla 4 se presentan los resultados por dimensión de los mismos y finalmente en la tabla 5 se responde a la variable correspondiente al objetivo general de la presente investigación. 
Tabla 4 Resumen total por dimensiones

Estadísticos descriptivos

\begin{tabular}{|l|c|c|c|c|c|}
\hline \multicolumn{1}{|c|}{ Dimensión } & N & Máximo & Mínimo & Promedio & $\begin{array}{c}\text { Desviación } \\
\text { estándar }\end{array}$ \\
\hline Emotividad & 97 & 3,67 &, 93 & 2,50 &, 46528 \\
\hline Eficacia & 97 & 4,00 &, 50 & 2,89 &, 53246 \\
\hline Pensamiento supersticioso & 97 & 3,62 &, 23 & 1,66 &, 66553 \\
\hline Rigidez & 97 & 3,10 &, 72 & 1,74 &, 47688 \\
\hline Pensamiento esotérico & 97 & 2,79 &, 29 & 1,54 &, 59373 \\
\hline Pensamiento ingenuo & 97 & 3,52 &, 90 & 2,52 &, 45109 \\
\hline
\end{tabular}

Fuente: Elaboración propia (2016)

Según el análisis estadístico referente a la primera dimensión Emotividad, se ha podido evidenciar que dentro de la población estudiantil de nuevo ingreso del Núcleo LUZ Costa Oriental del Lago existe un mayor promedio que los ubica en la categoría "alta presencia" 2,50 en la escala que integran: Autoestima, tolerancia, impasibilidad y capacidad de sobreponerse, lo que evidencia que son capaces de enfrentarse a las situaciones potencialmente estresantes tomándolas como un desafío y un aprendizaje, se describen como personas centradas, tranquilas, joviales y entusiastas, se aceptan tal y como son reflejando en sus acciones poseer tolerancia a la frustración ante los eventos imprevistos vividos. (García, 2005).

Para la segunda dimensión correspondiente a la Eficacia, según el análisis estadístico de cada uno de los indicadores: Pensamiento positivo, actividad y responsabilidad, de la población estudiantil de nuevo ingreso del Núcleo LUZ Costa Oriental del Lago se resume que existe un promedio de 2,89 en la categoría "Alta presencia".

Las personas con altas puntuaciones en eficacia son optimistas, entusiastas, enérgicos y fiables. Generalmente las personas con alta presencia de eficacia aceptan a los demás y no albergan rencores; más que juzgar a las personas trata de encontrar soluciones realistas a los problemas que se le presentan en su cotidianidad. (Epstein 2003).

Sobre los resultados correspondientes a la dimensión pensamiento supersticioso estos indican que las personas con este tipo de pensamiento creen que cuando sucede algo bueno después se compensará con algo malo o por el contrario pensar que si comenta algo que está pensando pasará algo malo y no se realizará. Las personas con estas creencias pasan todo el tiempo esperando que algo malo ocurra que disfrutando el presente, favoreciendo el pesimismo y la depresión. (Epstein 2003).

En cuanto a los resultados correspondientes a la dimensión Rigidez y sus tres indicadores, demuestran con 1,74 de promedio que los estudiantes tienen una mediana presencia de pensamientos y creencias rígidas extremas en el que no existen puntos de vistas medios sino es todo o nada, es blanco o negro ignorando las diferencias presentes en las personas y situaciones. 
Para la dimensión pensamiento esotérico indica que los estudiantes poseen una baja presencia de pensamientos y creencias paranormales e irracionales lo cual significa que no creen en fenómenos extraños, mágicos y científicamente cuestionables, tales como los fantasmas, la astrología, y las supersticiones convencionales.

Para la última dimensión del respectivo estudio se obtuvo unos resultados que indican una alta presencia de pensamiento ingenuo con un promedio de 2,52 que explican que las personas con altas puntuaciones en este tipo de pensamiento se asocian a una visión simplista de la vida y a una tendencia a evitar realidades desagradables y, a la vez, al desacierto por no tomar las debidas precauciones. Resultados que se ajustan a la etapa de la vida en la que se encuentran los estudiantes pertenecientes al estudio.

Tabla 5

Variable: Pensamiento Constructivo

\begin{tabular}{|c|c|}
\hline Promedio & 2,14 \\
\hline Categoria & Mediana presencia \\
\hline
\end{tabular}

\section{Discusión e Interpretación de los resultados Variable: Pensamiento constructivo}

Respondiendo al objetivo de la investigación en el que se plantea determinar el tipo de pensamiento presente en los estudiantes universitarios de nuevo ingreso del núcleo LUZ COL, los resultados presentados en la tabla 5 refieren que en las dos primeras escalas que corresponden a la emotividad y la eficacia hay una alta presencia que indican pensamientos constructivos, se aceptan a sí mismos y a los demás, también actúan con decisión en situaciones controlables y con escasa ansiedad en situaciones poco controlables.

Sin embargo en la escala que corresponde al pensamiento rígido se obtuvo una mediana presencia y una alta presencia relacionada con el pensamiento ingenuo lo que indica ciertos pensamientos desadaptativos y racionales con creencias básicas que determinan un modo de actuar sobre prejuicios e intolerancia así como la percepción de la vida de una manera simplista.

De manera que puede considerarse que los estudiantes pertenecientes a dicha investigación presentan una mediana presencia de pensamiento constructivo, esta variable se relaciona con comportamientos que estimulan el estrés, perciben los acontecimientos como más estresantes y el afrontamiento tanto mental como emocional es algunas veces desadaptativo, pudiendo traducirse en emociones angustiosas con elevados niveles de malestares físicos como dolores musculares, de cabeza y de estómago. (Epstein 2003).

Estos resultados obtenidos se corresponden con los presentados por Contreras, Chávez, Aragón y Velásquez (2010) quienes plantean que en el ámbito académico los alumnos que tienen 
muy bajo pensamiento constructivo, no logran desarrollar todo el potencial indicado por su coeficiente intelectual, posiblemente su incapacidad de pensar constructivamente interfiera con el uso de su capacidad intelectual. Concluyen los autores afirmando que un alumno que no logra relacionarse adecuadamente con sus maestros y compañeros, que mantiene una actitud derrotista, o que se agota psíquica e incluso físicamente por el estrés presentará desventajas en sus estudios.

\section{CONCLUSIONES Y RECOMENDACIONES}

Los resultados obtenidos y el análisis de la literatura científica permitieron presentar las siguientes conclusiones:

Tomando en cuenta que el objetivo general de la presente investigación es determinar el pensamiento constructivo bajo la visión de la inteligencia emocional en los estudiantes universitarios de nuevo ingreso del núcleo LUZ COL, con base a los resultados obtenidos se concluye que existe una mediana presencia relacionada con el pensamiento constructivo en los estudiantes lo cual indica que experimentan estrés con los cambios vividos, a veces son inflexibles y presentan un optimismo ingenuo con una visión simple de la vida, además suelen mostrar un retrato irreal de ellos mismos.

También se evidenció que los estudiantes perciben los acontecimientos y circunstancias de su existencia como hechos que les son difíciles de afrontar emocional y conductualmente, actitud propia de la edad en la que se encuentra la población en estudio, la adolescencia, etapa llena de variabilidad en cuanto al sistema de procesamiento de información que conforma el pensamiento en este periodo de la vida.

\section{Recomendaciones}

En correspondencia con los resultados obtenidos se presentan a continuación las siguientes recomendaciones:

- Dar a conocer los resultados del presente estudios a las autoridades de la universidad del Zulia núcleo costa oriental del lago, con el fin de planificar estrategias a través del departamento de Orientación sobre como potenciar el pensamiento constructivo en los estudiantes de nuevo ingreso que les permita desarrollar todo su potencial en el proceso de su carrera y vida universitaria.

- Desarrollar la presente investigación en otros contextos universitarios a fin de valorar las escalas de afrontamiento presente en los estudiantes. 


\section{REFERENCIAS BIBIOGRÁFICAS}

Ander-Egg, E. (2004) $8^{\circ}$ Técnicas de Investigación Social. Buenos Aires, Argentina: Hvmanitas.

Bisquerra R. (2011) Acción tutorial y educación emocional. 12 Congreso de Orientación Educativa. Guadalajara México. Julio México.

Contreras O., Chávez M., Aragón L., y Velásquez M. (2010) Estrategias de pensamiento constructivo en estudiantes universitarios. Universidad Nacional Autónoma de México. Revista Universitas psychologica Volumen 10 año $\mathrm{N}^{\circ} 1$ (pp.99-111).

Chávez M., Contreras O., y Velásquez M., (2014) Afrontamiento y depresión: su relación con pensamiento constructivo y no constructivo en universitarios. Revista Intercontinental de psicología y Educación volumen 16 Nº 2 (p.31-49).universidad Intercontinental México.

Epstein, S. (1998). Constructive thinking. The key of emotional intelligence.Londres: Paeger

Epstein S. (2012) Inventario de pensamiento constructivo: Una medida de inteligencia emocional. TEA

Ediciones S.A. Madrid. Documento en línea Disponible en http://www.web.teaediciones. com/Ejemplos/Manual_CTI.pdf consulta 18/12/2016

Epstein, S. (2003). Cognitive-experiential self-theory of personality. In Millon, T., \& Lerner.

Garaigordobil M. y Oñederra J. (2010) Inteligencia emocional en las víctimas de acoso escolar y en los agresores Revista European Journal of Education and Psychology volumen $3 \mathrm{~N}^{\circ} 2$ (pp243-256).

Hernández R., Fernández C. y Baptista L. (2014) Metodología de la Investigación (6ta. Ed.). México: McGrawHill.

Hurtado de Barrera, J. (2010). Metodología de la Investigación. Guía para la comprensión holística de la ciencia (4a. ed.). Caracas, Venezuela: Ediciones Quirón.

Martínez-Otero, V. (2002). "Reflexiones pedagógicas sobre la inteligencia", Pulso, Revista de Educación, $\mathrm{N}^{\circ} .25$, (pp. 77-86) 
Mayer J. y Salovey, P. (1990) Emotional Intelligence Imagination,Cognition and Personality, Número 9, (pp185-211).

Palella S. y Martins F. (2010) Metodología de Investigación cuantitativa. Tercera edición editorial FEDEUPEL. Venezuela.

Secretaría Docente Ingeniería LUZ-COL Venezuela.

Sierra R. (2005) Técnicas de investigación social: Teoría y ejercicios. Ediciones Paraninfo. Madrid. 